\title{
Probing the relaxed relaxion and Higgs portal scenarios with XENON1T scintillation and ionization data
}

\author{
Ranny Budnik, ${ }^{1,2, *}$ Hyungjin Kim, ${ }^{1, \dagger}$ Oleksii Matsedonskyi®${ }^{1, \$}$ Gilad Perez, ${ }^{1, \S}$ and Yotam Soreq $\odot^{3, \|}$ \\ ${ }^{1}$ Department of Particle Physics and Astrophysics, Weizmann Institute of Science, Rehovot 7610001, Israel \\ ${ }^{2}$ Simons Center for Geometry and Physics and C. N. Yang Institute for Theoretical Physics, \\ SUNY, Stony Brook, New York 11794, USA \\ ${ }^{3}$ Physics Department, Technion-Israel Institute of Technology, Haifa 3200003, Israel
}

(Received 21 February 2021; accepted 11 June 2021; published 7 July 2021)

\begin{abstract}
We study the recent XENON1T excess in the context of solar scalar, specifically in the framework of Higgs portal and the relaxion model. We show that $m_{\phi}=1.9 \mathrm{keV}$ and $g_{\phi e}=2.4 \times 10^{-14}$ can explain the observed excess in science run 1 (SR1) analysis in the 1-7 keV range. In the minimal scenarios we consider, the best-fit parameters are in tension with stellar cooling bounds. Despite this fact, the excess represents an example bringing attention to two interesting effects of general relevance. First, the scalarHiggs mixing angle reproducing the excess, $\sin \theta \simeq 10^{-8}$, is intriguingly close to the maximum value of mixing angle for the technical naturalness of the scalar mass. While finding a parameter value very close to its theoretical limit may naively seem an unlikely coincidence, we demonstrate that there exists a class of models which generically saturate the mixing naturalness bound. Secondly, we discuss a possibility that a large density of red giant stars may trigger a phase transition, resulting in a local scalar mass increase suppressing the stellar cooling. For the particular case of minimal relaxion scenarios, we find that such type of chameleon effects is automatically present but they can not ease the cooling bounds. They are however capable of triggering a catastrophic phase transition in the entire Universe. Following this observation we derive a new set of bounds on the relaxed relaxion parameter space. Finally, we present two nonminimal models that demonstrate how the cooling bounds can be relaxed as a result of high density effects.
\end{abstract}

DOI: 10.1103/PhysRevD.104.015012

\section{INTRODUCTION}

Recently, the XENON1T experiment reported an excess of electronic recoil events in the science run 1 (SR1) signal [1]. Within the energy range of $1-7 \mathrm{keV}$, the expected number of background only events is $232 \pm 15$, while the observed number of events is 285 with an apparent peak near $2-3 \mathrm{keV}$, in contrast to the expected flat background. The discrepancy corresponds to a $3.5 \sigma$ rejection of the background hypothesis in favor of an additional peaked spectrum resembling a solar axion source [1]. An unaccounted for background of tritium decay would lower the significance of the excess to about $2.2 \sigma$. While it is possible that the excess is due to a statistical fluctuation or yet

ran.budnik@weizmann.ac.il

†hyungjin.kim@weizmann.ac.il

‡oleksii.matsedonskyi@weizmann.ac.il

§ilad.perez@weizmann.ac.il

"soreqy@physics.technion.ac.il

Published by the American Physical Society under the terms of the Creative Commons Attribution 4.0 International license. Further distribution of this work must maintain attribution to the author(s) and the published article's title, journal citation, and DOI. Funded by SCOAP. another unaccounted background, we focus on the case that it is due to the existence of a new degree of freedom with a mass smaller than a few $\mathrm{keV}$.

The interpretation for the excess as a solar axion with $m_{a} \lesssim$ $0.1 \mathrm{keV}$ leads to an electronic coupling of $g_{a e} \sim 3 \times 10^{-12}$, where the corresponding upper bound is $g_{a e}<3.7 \times 10^{-12}$ [1]. This is consistent with the LUX solar axion search, which implies an upper bound of $g_{a e}<3.5 \times 10^{-12}$ [2], but in tension with astrophysical bounds from stellar cooling. Reference [3] reported an upper bound of $g_{a e} \lesssim 3 \times 10^{-13}$ from red giant (RG) stars cooling. Yet there are hints for a signal in anomalous energy loss in white dwarfs, RG stars, and neutron stars which point to a preferred coupling of $g_{a e}=(1.6 \pm 0.3) \times 10^{-13}$ [4] (see also [5]). However, as pointed by Ref. [6], a light scalar with a mass at or below the $\mathrm{keV}$ scale can be produced in the Sun and be probed by dark matter (DM) direct detection experiments through electron ionization at the $\mathrm{keV}$ scale. In this work we mainly focus on this possibility and confront it with the XENON1T excess. Other possible implications of the recent XENON1T data were discussed in [7-33].

Producing a light scalar (or a pseudoscalar with $C P$-odd couplings) is generically a nontrivial task from the modelbuilding point of view. We will concentrate on two cases: a 
generic scalar Higgs portal scenario, which can be seen as an effective description of various more complicated constructions, and a more predictive relaxion model [34] motivated by the Higgs mass naturalness problem. While the relaxion is considered to be a pseudoscalar, its vacuum generically breaks $C P[35,36]$ leading to a scalarlike phenomenology.

Below, we analyze the recent science run 1 XENON1T result [1] in the context of a new scalar field with mass at the $\mathrm{keV}$ scale or below and show that such a new particle is compatible with the excess. In addition, we explore its implications for the S2-only analysis [37] and show that such scalar with $\mathrm{keV}$ mass has a clear signature in terms of a bump on flat background. However, the bounds from stellar cooling are stronger [38] and exclude the preferred region of the parameter space. We also consider the case where the tritium background is taken into account and show that the preferred parameter space is consistent with a smaller coupling and the tension with stellar cooling bound is weakened.

Finally, we map the relevant parameter space to the generic Higgs portal and the relaxion model [34]. We show that the reported excess is located close to the naturalness bound of the scalar-Higgs mixing angle and argue that such a feature is in fact generic for a certain class of scenarios. We also discuss a possibility that a localized phase transition takes place inside RGs, ${ }^{1}$ locally increasing the scalar mass, and, hence, alleviating the tension with stellar cooling bounds. We find that such phase transition can occur in minimal (non-QCD) relaxion models, but, unfortunately, in a region of parameter space not compatible with the reported excess. Nevertheless, we derive a new constraint on the relaxion parameter space, which is required to avoid a new phase, with unrealistic Higgs mass, to fill the whole Universe.

\section{THE SOLAR RELAXION/SCALAR SIGNAL}

We estimate the solar scalar signal by following Ref. [6]. For the axion case, see [39]. The relevant $\phi$-electron interaction Lagrangian is given by

$$
\mathcal{L} \supset-g_{\phi e} \phi \bar{e} e .
$$

Below, we focus on the $m_{\phi} \lesssim 3 \mathrm{keV}$ mass range and consider finite mass effects.

Within the Sun, light scalars can be produced by various production mechanisms: bounded electrons (bb), recombination of free electrons (bf), Bremsstrahlung emission due to scatterings of electrons on ions (ff), Bremsstrahlung emission due to scatterings of two electrons (ee), and Compton-like processes (C). At the relevant energy scale,

\footnotetext{
${ }^{1}$ While the horizontal branch stars cooling is also in conflict with the observed excess, we first focus on the stronger tension coming from RGs.
}

the dominant production rate is the electron-ion Bremsstrahlung. The total differential scalar flux is estimated as

$$
\frac{d \Phi}{d \omega} \approx \frac{\omega k}{8 \pi^{3} R^{2}} \int_{\odot} d V \Gamma^{\text {prod }}(\omega)
$$

where $\Gamma^{\text {prod }}$ is sum over all production rates, $R=1 \mathrm{AU}$ is the distance between the Earth and the Sun, $\omega$ and $k$ are the scalar energy and momentum, respectively, and $V$ is the Sun volume, where the Sun profile is taken from [40].

The ratio between the matrix elements of a $\gamma$ emission and a $\phi$ emission (or absorption) is given by [41]

$$
\frac{|\mathcal{M}(e \rightarrow e \phi)|^{2}}{|\mathcal{M}(e \rightarrow e \gamma)|^{2}} \approx \frac{g_{\phi e}^{2}}{4 \pi \alpha} \beta^{2}
$$

where $\beta=k / \omega$ is the scalar velocity. Since the ratio in Eq. (3) enters both in the production and in the detection (divided by $\beta$ ), the ratio between the number of scalar and pseudoscalar events rates can be written as

$$
\frac{\mathcal{R}_{\phi}(\omega)}{\mathcal{R}_{a}(\omega)}=\frac{g_{\phi e}^{4}}{g_{a e}^{4}} \frac{m_{e}^{4}}{\omega^{4}}\left(4 \frac{\beta^{2}}{3-\beta^{2 / 3}}\right)^{2}
$$

where $\mathcal{R}_{\phi, a}(\omega)=\frac{d \Phi}{d \omega} \sigma_{\phi, a}(\omega)$, and $\sigma_{\phi, a}(\omega)$ is scalar and pseudoscalar absorption cross-section for liquid Xenon [6,41-43]. From Eq. (4) we learn that the solar scalar signal is softer than the solar axionlike case, and, thus, it will be peaked at lower energies.

Next, following [6] we evaluate the $\mathcal{R}_{\phi}(\omega) \epsilon_{\mathrm{Xe}}$, where the XENON1T detector efficiency, $\epsilon_{\mathrm{Xe}}$, is taken from [1]. The detector effects are taken into account by a Gaussian smearing of the signal, where the relevant parameters are adopted from [44]. The predicted $\phi$ event rates (after smearing) for three benchmark points, $\mathrm{BM}_{1,2,3}$, with $m_{\phi}=$ $(0,1.3,1.9) \mathrm{keV}$ and $g_{\phi e}=(0.8,1.5,2.4) \times 10^{-14}$, respectively, are plotted in Fig. 1. We validated the smearing procedure by smearing the massless axion signal spectrum from Ref. [39] and comparing it to Fig. 1 of [1], and found a matching up to few percent level.

In addition to the above signal, manifested in both a scintillation signal (S1) and an ionization signal (S2), we consider the scalar signal in the XENON1T's S2-only analysis [37], where the energy threshold is lower, $\sim 200 \mathrm{eV}$. Since in the scalar case the signal is softer, it is expected to have a better sensitivity in the S2-only analysis. Scalars with masses around the solar interior plasma frequencies, $1 \mathrm{eV} \lesssim \omega_{\mathrm{p}} \lesssim 300 \mathrm{eV}$, have enhanced production rate due to mixing with the photon longitudinal mode in the Sun plasma [38]. As pointed out in [6], the resulting sensitivity for $m_{\phi} \lesssim 300 \mathrm{eV}$ by using the XENON1T S2-only dataset [37] is improved by an order of magnitude. This resonant production is only efficient for 


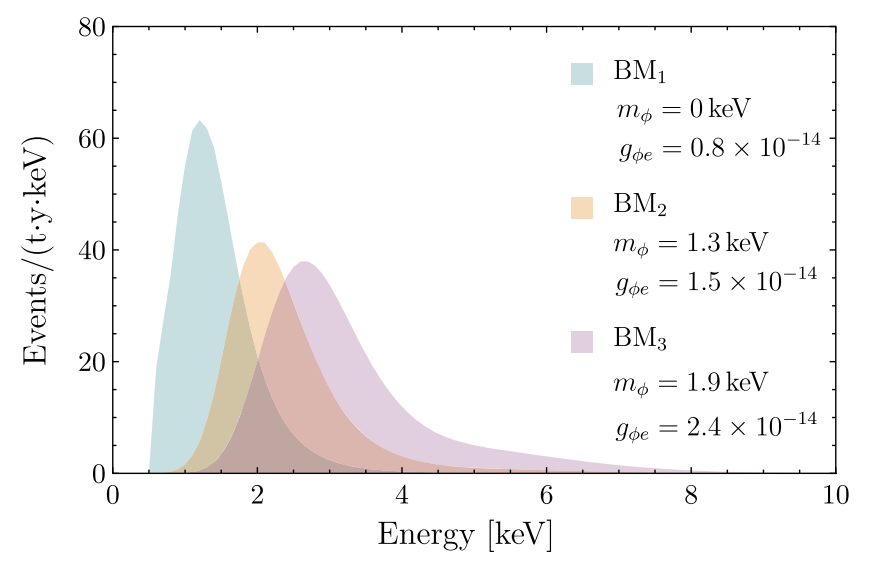

FIG. 1. The solar scalar event rates are shown for three benchmark points as indicated on the plot. The shown event rates include the detector efficiency and resolution. See the main text for details.

scalar masses below the local plasma frequency, which affects the shape of the expected spectrum with respect to the scalar mass. We finally note that in-medium mixing effect at the detector is negligible for the solar scalar, while it could be important for direct detection experiments for light scalar dark matter [45].

\section{RECASTING OF THE XENON1T EXCESS AS A RELAXION/SCALAR}

We fit the scalar signal for the SR1 dataset of Ref. [1] with and without the tritium background as follows. In the first case, we construct a likelihood function of $m_{\phi}$ and $g_{\phi e}$ for the scalar signal and background. We take the background model as fixed, directly from Fig. 4 of [1]. This is justified as the background (without the tritium) is essentially fixed by the high energy spectrum and the injection of the signal at low energy has negligible effect on it. This is

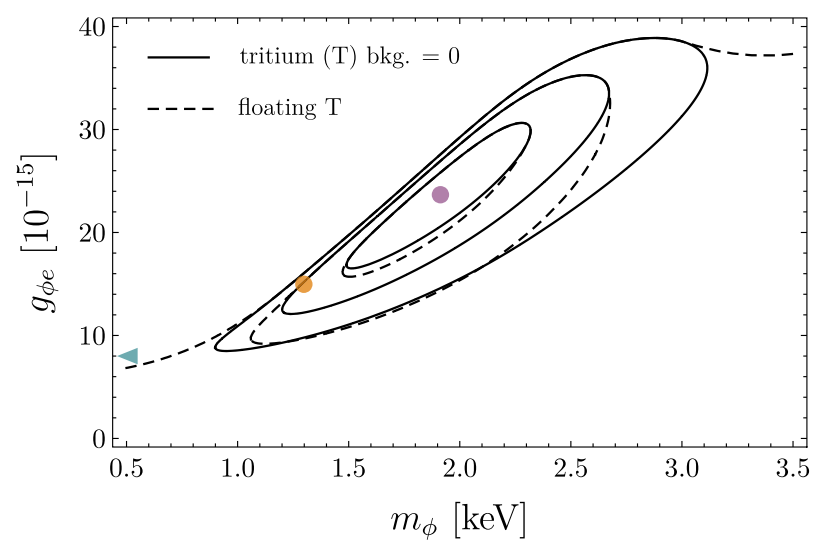

evident from the $\sim 2 \%$ change at the high end energy tail in the best fit while considering the solar axion, tritium, and $\nu$ magnetic moment in Ref. [1]. To assess the sensitivity of the result, we also add the tritium background component, where we profile over its magnitude.

By minimizing the likelihood, the best-fit point (with and without the tritium background) is found to be $m_{\phi}=$ $1.9 \mathrm{keV}$ and $g_{\phi e}=2.4 \times 10^{-14}$, where the left panel of Fig. 2 shows the $68 \%, 95 \%$, and $99 \%$ confidence intervals in the $m_{\phi}-g_{\phi e}$ plane with and without the tritium background. To find the contours we apply the asymptotic formula from [46] for two free parameters. We find that the preferred region is for a finite $m_{\phi} \sim 2 \mathrm{keV}$. This is in contrast to the pseudoscalar case, where an effectively massless solution is favored. The reason is that a massless or very light scalar spectrum has a significant soft component relative to the pseudoscalar case, as emphasized in Eq. (4). The right panel of Fig. 2 demonstrates this point, showing a comparison between the signal and background with respect to the XENON1T data for the three benchmark models, $\mathrm{BM}_{1,2,3}$. We note that the preferred region in the parameter space is in tension with the upper bound found from limits on RG cooling including plasmon-scalar mixing effect, $g_{\phi e}<7 \times 10^{-16}$ [38]. As a cross check, we have performed the same likelihood analysis for the pseudoscalar case and found good agreement with the result of [1].

In addition to the S1 and S2 signal, we now consider the possibility of a scalar signal in the S2-only analysis of XENON1T [37]. This analysis only uses a partial background model, making possible setting upper bounds on signals and testing the consistency of a given signal. In Fig. 3, we plot the S2-only expected signal for $\mathrm{BM}_{2}$, $m_{\phi}=1.3 \mathrm{keV}$ and $g_{\phi e}=1.5 \times 10^{-14}$, and compare it to the expected background and the data from [37]. For the purpose of demonstration, we have multiplied the signal by

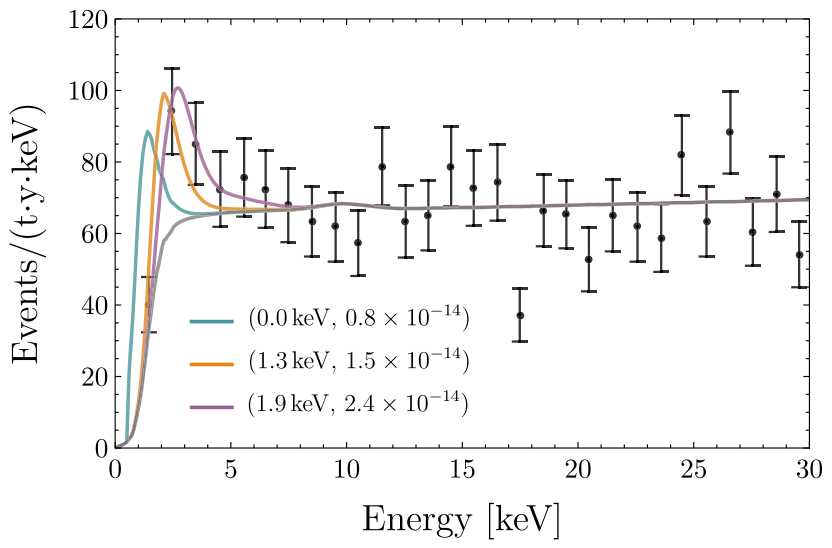

FIG. 2. Left: The $68 \%, 95 \%$, and $99 \%$ confidence intervals are shown, solid (dashed) contours are with (without) the tritium background. The three benchmark points with $m_{\phi}=(0,1.3,1.9) \mathrm{keV}$ and $g_{\phi e}=(0.8,1.5,2.4) \times 10^{-14}$ are marked in cyan, orange, and purple, respectively. The purple $\left(\mathrm{BM}_{3}\right)$ is the best-fit point. Right: The signal + background is shown for the three benchmark points. The black points and gray line are data and background (without tritium) from [1], respectively. 


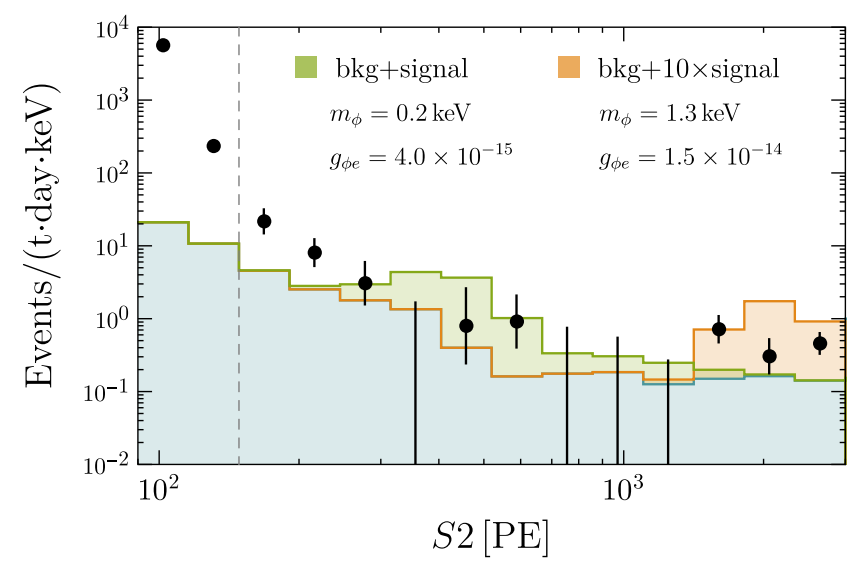

FIG. 3. The signal and background for the S2-only analysis is shown. The $\mathrm{BM}_{2}$ (orange), $m_{\phi}=1.3 \mathrm{keV}$ and $g_{\phi e}=1.5 \times 10^{-14}$, is chosen for this plot. The signal is enhanced by 10 for the illustrative purpose. In addition, we have also plotted the signal that would arise from $m_{\phi}=200 \mathrm{eV}$ and $g_{\phi e}=4 \times 10^{-15}$ (green). Here, the coupling constant is chosen to show the spectrum of events, although it is only marginally consistent with the current S2-only analysis [6]. Also, for this choice of mass, the event spectrum for SR1 excess is more or less similar to $\mathrm{BM}_{1}$ for the same coupling constant. See the main text, especially Sec. V, for the potentially interesting phenomenological consequences related to this choice of parameters.

10. We have also verified that the best-fit parameters for SR1 dataset of Ref. [37] is consistent with S2-only analysis. In addition to $\mathrm{BM}_{2}$, we have also plotted the signals of $m_{\phi}=200 \mathrm{eV}$ and $g_{\phi e}=4 \times 10^{-15}$. For these parameters, the spectral shape of events for SR1 excess is close to the $\mathrm{BM}_{1}$ in Fig. 2, while the events at the peak are suppressed by less than ten percent for the same coupling constant. This choice of parameters, especially in the context of relaxed relaxion, may lead to interesting phenomenological consequences inside stellar objects due to finite density corrections to the potential. This will be briefly discussed in Sec. V.

\section{NATURALNESS MIRACLE}

We will now confront the observed excess of events with theoretical models. Let us start with the case of a generic scalar Higgs portal model, containing one new scalar degree of freedom. Its coupling to the electrons comes from the mixing with the Higgs and is given by

$$
g_{\phi e}=\frac{\lambda_{e}}{\sqrt{2}} \sin \theta
$$

where $\lambda_{e}$ is the electron Yukawa, and $\sin \theta$ is the mixing whose best-fit value turns out to be $\sin \theta \simeq 1.2 \times 10^{-8}$. In the absence of any special cosmological dynamics, naturalness implies an upper bound on the $\phi-h$ mixing angle (the red line in Fig. 4) [47-49] (see also Appendix):

$$
\sin \theta<\frac{m_{\phi}}{m_{h}} \simeq 1.5 \times 10^{-8}\left(\frac{m_{\phi}}{1.9 \mathrm{keV}}\right)
$$

where in the last equality we used the best-fit value for the scalar mass and $m_{h} \approx 125 \mathrm{GeV}$ is the Higgs mass. While the best-fit mixing satisfies the naturalness bound, it appears to be strikingly close to the boundary of the natural region. Below we will argue that in fact, in Higgs portal models, having a mixing close to the naturalness bound is a generic feature which does not require any fine adjustments. We will here follow a very simplistic line of arguments, while for more details we refer the reader to the Appendix.

Let us consider a Higgs portal potential, with the only Higgs- $\phi$ interaction given by a term $\mu|H|^{2} \phi$. Because of the resulting mass mixing, the scalar $\phi$ inherits the Higgs couplings, suppressed by the mixing angle

$$
\sin \theta \simeq \mu / v,
$$

where $v$ is a Higgs VEV. We also find that the physical $\phi$ mass at $\phi=0$ is modified by the presence of the mixing

$$
m_{\phi}^{2}=m_{0}^{2}-m_{h}^{2} \sin ^{2} \theta
$$

where $m_{0}^{2}$ is the mixing-independent part of $\phi$ mass. The XENON1T excess corresponds to $m_{\phi}^{2} \simeq m_{h}^{2} \sin ^{2} \theta$. Such a relation can be reproduced for $m_{0}^{2} \simeq 2 m_{h}^{2} \sin ^{2} \theta$, requiring a coincidence of scales $m_{0} \sim \mu$. The naturalness bound can however also be saturated for any $m_{0}^{2} \ll m_{h}^{2} \sin ^{2} \theta$, corresponding to $m_{0} \ll \mu$, and thus no need to equate $a$ priori unrelated parameters. This happens because for large $\mu$, the mass term at $\phi=0$ is negative, and the actual minimum (when formed by the interplay of negative quadratic and positive cubic or quartic term) is characterized by the physical mass $m_{\phi}^{2} \simeq m_{h}^{2} \sin ^{2} \theta$. This means that any point on the naturalness line can be realized in multiple ways, which feature almost identical $\mu$ parameters, but different $m_{0}$ such that $m_{0} \ll \mu$.

As was already mentioned, the best-fit value of the $g_{\phi e}$ coupling is in tension with the stellar cooling bounds. In the relevant mass range, the strongest constraints are derived from the RGs evolution [38], $g_{\phi e} \lesssim 10^{-15}$, and are valid for the scalar masses $\lesssim 20 \mathrm{keV}$. Such bounds however can be avoided assuming the properties of the $\phi$ field are modified in the dense interior of RG stars. The RG core density significantly exceeds that of the Sun, reaching the nucleon and electron number density $n_{\mathrm{RG}} \sim 10^{15} \mathrm{eV}^{3}$ [38,50], and in principle can affect the local scalar mass, making the cooling bound inapplicable. This can be realized for instance if the $\phi$ field potential is characterized by two minima, one being the true minimum in the vacuum, and another becoming the energetically preferred state inside the RG stars, as a result of a correction to the scalar 
potential $\delta V \simeq g_{\phi N} n_{\mathrm{RG}} \phi$, where $g_{\phi N}$ is a nucleon-scalar coupling. These two minima have to be characterized by significantly different masses. Constructing a potential satisfying all the aforementioned criteria is however a very nontrivial task, which we leave beyond the scope of the current letter.

\section{THE RELAXED RELAXION CASE}

Relaxion mechanism [34] allows to explain the smallness of the Higgs mass by nontrivial cosmological dynamics of the Higgs relaxion system. As was pointed out in [51], around its first minima, the shape of the relaxion potential is very shallow and highly nonsymmetric unlike the generic potentials of pseudo-Nambu-Goldstone bosons, which leads to several interesting phenomenological implications relevant for accelerators and tabletop experiments. Here we discuss additional implications of the relaxion's exotic potential. We begin with just describing the relevant properties of the relaxed relaxion and then move to the implications. The relaxion mass and electron coupling are predicted [51]

$$
m_{\phi}^{2} \simeq \frac{\Lambda_{\mathrm{br}}^{4}}{f^{2}} \frac{\Lambda_{\mathrm{br}}^{2}}{\Lambda v}, \quad g_{\phi e}=\frac{\lambda_{e}}{\sqrt{2}} \sin \theta \simeq \lambda_{e} \frac{\Lambda_{\mathrm{br}}^{4}}{f v^{3}},
$$

where $\Lambda$ is the cutoff, $f$ and $\Lambda_{\mathrm{br}}$ are the period and amplitude of Higgs-dependent barriers, $\theta$ is the relaxionHiggs mixing angle, and $v=174 \mathrm{GeV}$ is the Higgs VEV. Note that the formulas above are only order of magnitude estimates. Assuming $f=\Lambda$ and the Standard Model (SM) value for the electron Yukawa coupling $\lambda_{e}=\lambda_{e \mathrm{SM}}$, we find for the best-fit values that

$$
\Lambda=f \simeq 60 \mathrm{TeV}, \quad \Lambda_{\mathrm{br}} \simeq 10 \mathrm{GeV} .
$$

More generally, for $f>\Lambda$ we have a continuum of possibilities, allowing for $\Lambda<60 \mathrm{TeV}<f$ and $\Lambda_{\mathrm{br}}>10 \mathrm{GeV}$. Furthermore, for $f=\Lambda$, the order of magnitude of the inflationary Hubble parameter is constrained to be within $1 \mathrm{eV}$ and $0.1 \mathrm{GeV}$. In Fig. 4 we show the position of the excess in the allowed parameter space of the relaxion models (green band), together with relevant experimental bounds.

For the best-fit mass, the relaxion model implies the relaxion-Higgs mixing angle is within the range of $\sin \theta \in$ $\left[10^{-10}, 10^{-5}\right][51]$; see Fig. 4. Thus, by relaxing the assumption of $\lambda_{e}=\lambda_{e S M}$, we can identify a preferred range for the electron Yukawa to be $10^{-3}<\lambda_{e} / \lambda_{e S M}<10^{2}$, which is consistent with the current direct upper bound of $\lambda_{e} \lesssim 600 \times \lambda_{e \text { SM }}$ [62-64].

Let us now discuss whether the relaxion mechanism can overcome the stellar cooling bounds with a help of the chameleon effect discussed in the previous section. ${ }^{2}$

\footnotetext{
${ }^{2}$ Density effects on light particles were also considered in other contexts; see e.g., $[65,66]$.
}

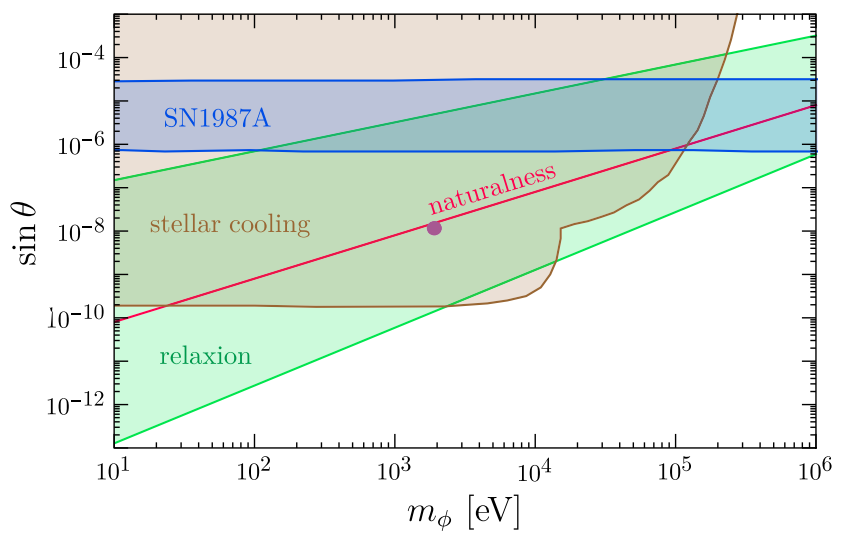

FIG. 4. The best fit to XENON1T excess (purple dot) in terms of the mixing angle, $\sin \theta$, and the scalar mass, $m_{\phi}$, together with the constraints from the stellar cooling [38,52,53] (brown), SN1987A [54-56] (blue), as well as preferred relaxion parameter space [51] (green), and the naturalness bound (red). We note that the SN1987A constraint depends on SNe explosion mechanism [57-61].

Potential importance of the density effects inside of neutron stars on the QCD relaxion was already emphasized in Ref. [67], while here we concentrate on the non-QCD version of the relaxion mechanism. The relaxion potential naturally features a set of consecutive minima, and may travel between them if the density-induced relaxion field displacement is large enough. In the minimal relaxion scenario, the local nucleon number density $n$ induces a linear piece in the potential $\delta V \simeq g_{\phi N} n \phi$ which shifts the relaxion in the direction of the next deeper minimum. For a sufficiently large shift the relaxion will start rolling towards the next minimum. However, in the absence of efficient friction $^{3}$ and with negligible gradient energy, we expect that the relaxion will not stop until it reaches the global minimum of its potential, featuring a large negative Higgs mass squared of the order of the cutoff scale $\Lambda$. If the large density region is larger than the critical bubble, the new phase will expand outside and fill the Universe. Otherwise, localized bubbles [75,76] within the dense astrophysical objects will be formed.

To induce such a phase transition (PT), the densityinduced relaxion field displacement, $\delta \phi_{n} \simeq \delta V^{\prime} / m_{\phi}^{2}=$ $g_{\phi N} n / m_{\phi}^{2}$, has to exceed the distance between the minimum and the closest maximum of the relaxion potential, given by $\Delta \phi \simeq \Lambda_{\mathrm{br}}^{2} f / \Lambda v$ [51]. Using Eq. (9) we find that the transition requires

$$
\frac{\delta \phi_{n}}{\Delta \phi} \simeq \frac{g_{\phi N} n \Lambda^{2}}{v \Lambda_{\mathrm{br}}^{4}} \gtrsim 1,
$$

and it will expand outside of the dense object if the object's radius $R_{*}$ is greater than the critical bubble radius which we approximately estimate as $1 / m_{\phi}$ :

\footnotetext{
${ }^{3}$ See $[68-74]$ for a possible friction source.
} 


$$
R_{*} \gg 1 / m_{\phi}
$$

An existence of localized phases not satisfying the latter condition is an interesting topic which we leave for future studies.

On the other hand, the scenario with the wrong Higgs VEV bubbles expanding outwards is excluded experimentally. It is important to find out how this fact limits the size of $\sin \theta$ which has a paramount importance for the relaxion experimental tests. Expressing Eq. (11) through $m_{\phi}, \sin \theta$, and $\Lambda$,

$$
\frac{\delta \phi_{n}}{\Delta \phi} \simeq \frac{g_{\phi N} n m_{\phi}^{4} \Lambda^{4}}{\sin ^{4} \theta v^{11}} \gtrsim 1
$$

we see that there exists some minimal value of $\sin \theta$, below which the PT always happens, and it is given by

$$
\sin \theta_{\min } \simeq \frac{\left(g_{\phi N} n\right)^{1 / 4} m_{\phi} \Lambda}{v^{11 / 4}} .
$$

The absolute lower bound on the mixing is then proportional to the lower bound on the cutoff scale $\Lambda$, for which we take $1 \mathrm{TeV}$.

In Fig. 5 we demonstrate our findings, applied to neutron stars, RGs and the Sun. We assume the minimal coupling $g_{\phi N}=g_{h N} \sin \theta$, where $g_{h N} \sim 10^{-3}$ is the Higgs coupling to nucleons. Colored areas show where the transition always happens and propagates outside the dense objects. Such PT can also happen for larger $\sin \theta$ for some parameter choices. For masses lower than the inverse size of the corresponding astrophysical objects (left to corresponding colored areas) the PT can happen, but it is localized within the dense objects. For this plot we only chose to show the bounds from three distinct types of high-density astrophysical

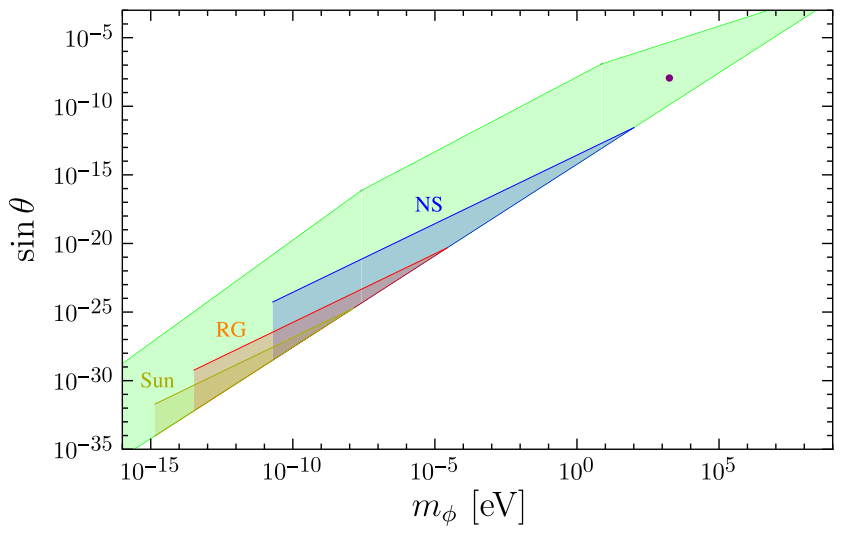

FIG. 5. Relaxion parameter space in the first minimum (green) in terms of $\sin \theta$ and $m_{\phi}$. Purple dot shows the best fit to XENON1T excess. The blue, red, and yellow regions show where the expanding bubbles are produced, resulting from neutron stars, RGs cores, and the Sun core, respectively. See text for more details. bodies, not aiming at a comprehensive analysis of all possible stars.

Let us briefly comment on how the plot in Fig. 5 would change if we chose a different minimal cutoff $\Lambda$ which is set to $1 \mathrm{TeV}$ here. Each of the points in the bulk of the green band and the excluded regions can be realized by a continuum of $\Lambda$ values, but some of the boundaries of these regions are directly sensitive to the minimal cutoff $\Lambda$. In particular, for increased minimal $\Lambda$, the lower edge of the relaxion band will go up, with $\sin \theta_{\min } \propto \Lambda_{\min }$, see [51]. At the same time, the minimal value of the mixing, below which the transition always happens, is also $\propto \Lambda_{\min }$; see Eq. (14). Therefore, in the log plot, the unstable regions will shift up with increase of the minimal cutoff, while the green band will shrink from below. Besides, the upper bound of the relaxion band is quadratically sensitive to the maximal allowed value of $\Lambda_{\mathrm{br}}$ which in this case is set to $v$.

As one can see from the plot, the RG-localized PT region is located, as trivially expected, far away from the XENON1T excess point not allowing to reconcile the latter with the stellar cooling.

Notice that the derived bounds can be substantially changed assuming (nonminimal) stronger relaxion coupling to nucleons. The current experimental bound on proton coupling is $g_{\phi p} \lesssim 10^{-6}$ for $m_{\phi} \lesssim 0.1 \mathrm{keV}$ [77] (for stronger bounds on coupling to neutrons see [78-81]). Such an increased coupling can not help with resolving the stellar cooling tension.

\section{TWO FIELD MODELS TO RELAX THE COOLING BOUNDS}

We now present two simplistic models allowing to locally increase the mass of the scalar $\phi$ to the value above $\sim 20 \mathrm{keV}$ inside of red giants and horizontal branch (HB) stars. The effective induced potential of the $\phi$ field takes the form

$$
V(\phi)=\frac{1}{2} m_{\phi}^{2} \phi^{2}+\frac{1}{4} \lambda_{\phi} \phi^{4}+\frac{n^{p} \phi^{q}}{F^{3 p+q-4}}+g_{\phi e} \phi \bar{e} e,
$$

where $F$ is some mass scale. The $\phi$-electron coupling is fixed to reproduce the excess. In the following we will restrict to $p=1,2$ and to the situations where the third term of Eq. (15), sensitive to the matter density, increases the mass of the $\phi$ field without changing the position of the minimum, hence $q=2$. To reproduce the excess, we require

$$
\begin{aligned}
m_{\phi, \text { Sun }} & \simeq 1.9 \mathrm{keV}, \\
m_{\phi, \mathrm{HB}, \mathrm{RG}} & >20 \mathrm{keV},
\end{aligned}
$$

where $m_{\phi, X}$ is the $\phi$ effective mass in the environment $X$. The last requirement removes the tension with $\mathrm{HB}$ and $\mathrm{RG}$ cooling; see Fig. 4.

As we will show later, this model requires to be completed with new physics below some of the relevant 
$\phi$ effective masses, in which case the potential (15) can not be attributed to an effective field theory (EFT). It can be obtained from a potential with additional light scalar field $\chi$,

$V(\phi, \chi)=\frac{1}{2} m_{\phi}^{2} \phi^{2}+\frac{1}{4} \lambda_{\phi} \phi^{4}+\frac{1}{2} m_{\chi}^{2} \chi^{2}+g_{\chi N} n \chi+\kappa \chi^{\alpha} \phi^{\beta}$.

Shifting the $\chi$ field origin to the new minimum inside of dense objects, $\chi \rightarrow \chi-g_{\chi N} n / m_{\chi}^{2}$, the effective induced potential for $\phi(15)$ is obtained.

The potential of Eq. (15) is reproduced from the one of (17) for $\{p, q\}=\{\alpha, \beta\}=\{1,2\},\{2,2\}$. The $\{2,2\}$ case corresponds to the leading order expansion of the potential of Ref. [82] designed for the same purpose. Let us consider the two cases in detail.

\section{A. Model with $\{p, q\}=\{1,2\}$}

We chose the following parameters of the reduced potential (15):

$$
m_{\phi}=0.5 \mathrm{keV}, \quad F=-\frac{m_{\chi}^{2}}{\kappa g_{\chi N}}=0.1 \mathrm{MeV},
$$

which give

$$
\begin{aligned}
m_{\phi, \text { Sun }} & \simeq 1.9 \mathrm{keV}, \quad m_{\phi, \mathrm{HB}} \simeq 30 \mathrm{keV}, \\
m_{\phi, \mathrm{RG}} & \simeq 0.3 \mathrm{MeV} .
\end{aligned}
$$

Besides the conditions (16), we also need to satisfy the star cooling bounds on $\phi-N$ interactions. While the large effective mass in RG removes the corresponding bound, in $\mathrm{HB}$ we obtain, recasting the results of [38], the following estimate of the bound:

$$
\frac{1}{4 \pi}\left[\frac{g_{\chi N} \kappa E}{E^{2}+m_{\chi}^{2}}\right] \lesssim 10^{-10}
$$

The lhs of Eq. (20) accounts for the $\chi$ propagator necessary to produce two $\phi$ quanta from plasma, phase space suppression for two-body decay, and we will take $E \sim$ $m_{\phi, \mathrm{HB}}$ for the typical energy scale in the problem. For $g_{\chi N} \kappa / m_{\chi}^{2}=F$ fixed by (18), this leads to

$$
m_{\chi}^{2} \lesssim 10^{-9} F m_{\phi, \mathrm{HB}} \simeq(1 \mathrm{eV})^{2} .
$$

A set of parameters, satisfying the above inequality and the fifth force bounds on $\chi-N$ interactions, is for instance

$$
m_{\chi}=10^{-8} \mathrm{eV}, \quad g_{\chi N}=10^{-22}, \quad \kappa=-10 \mathrm{eV} .
$$

Furthermore, the presence of the cubic coupling between $\phi$ and $\chi$ induces an instability of the potential. We compute the corresponding tunneling action in vacuum, assuming that tunneling happens along a straight trajectory $t$ in $\phi-\chi$ plane, and scan over all possible $t$ directions passing through $V(\phi, \chi)<0$, thereby finding the trajectory with the minimal action. Using the exact solution for quadratic plus cubic potential [83], $S_{23} \simeq 205 m_{t}^{2} / \kappa_{t}^{2}$ (where $m_{t}$ and $\kappa_{t}$ are the mass and the cubic coupling in a $t$ direction), we found $\min \left[S_{23}\right] \simeq 10^{9}$. Using the thick wall approximation [84], $S_{\text {thick wall }} \simeq \pi^{2}\left(\phi_{\text {exit }}^{2}+\chi_{\text {exit }}^{2}\right)^{2} /\left|V_{\text {exit }}\right|$, and scanning over all possible exit points, we obtained $\min \left[S_{\text {thick wall }}\right] \simeq 10^{8}$. Both numbers correspond to the tunneling being sufficiently suppressed. Also notice that inside of dense objects the tunneling is expected to be even more suppressed, as the density effect shifts the $\chi$ minimum in the direction opposite to the tunneling direction.

Another option to relax the cooling bounds could be to further decrease the scale $F$, leading to $m_{\phi, \mathrm{HB}} \gtrsim 0.2 \mathrm{MeV}$ where the HB cooling bound disappears. In practice, this leads to a too large $\phi$ effective mass in the Sun.

\section{B. Model with $\{\boldsymbol{p}, \boldsymbol{q}\}=\{\mathbf{2}, \mathbf{2}\}$}

We assume that the cooling bounds on $\phi-N$ interaction are significantly relaxed in this case and can be ignored. We choose the following parameters for the reduced potential:

$$
m_{\phi}=0.5 \mathrm{keV}, \quad F=\frac{m_{\chi}}{\kappa^{1 / 4} g_{\chi N}^{1 / 2}}=50 \mathrm{keV},
$$

giving

$m_{\phi, \text { Sun }} \simeq 1.9 \mathrm{keV}, \quad m_{\phi, \mathrm{HB}} \simeq 24 \mathrm{keV}, \quad m_{\phi, \mathrm{RG}} \simeq 2.4 \mathrm{MeV}$,

Such a low value of $F$ again does not allow to consider the model with only one field $\phi$ as an EFT. The extended potential with the $\chi$ field can then be defined for instance by

$m_{\chi}=10^{-7} \mathrm{eV}, \quad g_{\chi N}=10^{-22}, \quad \kappa=10^{-3}$.

This concludes our discussion of the two-field models.

\section{ACKNOWLEDGMENTS}

We would like to thank Kfir Blum, Diego Redigolo, and Tomer Volansky for fruitful discussions, and Abhishek Banerjee for technical support. We are grateful to $\mathrm{Oz}$ Davidi for his contributions to this project in its initial phase. The work of R. B. is supported by ISF Grant No. 1937/12. R. B. is the incumbent of the Arye and Ido Dissentshik Career Development Chair. The work of O. M. is supported by the Foreign Postdoctoral Fellowship Program of the Israel Academy of Sciences and Humanities. The work of G. P. is supported by grants from The U.S.- Israel Binational Science Foundation (BSF), 
Israel Science Foundation (ISF), German Israeli Foundation (GIF), Yeda-Sela-SABRA-WRC, and the Segre and the Friedrich Wilhelm Bessel Research Awards. Y. S. is supported by the BSF (NSF-BSF program Grant No. 2018683) and by the Azrieli Foundation. Y. S. is a Taub fellow (supported by the Taub Family Foundation).

\section{APPENDIX: NATURALNESS BOUND AND ITS SATURATION}

Let us consider generic renormalizable scalar potential

$$
\begin{aligned}
V(h, \phi)= & \frac{1}{2} \mu h^{2} \phi+\frac{1}{4} \lambda_{h \phi} h^{2} \phi^{2} \\
& -\frac{1}{2} m_{h 0}^{2} h^{2}+\frac{1}{4} \lambda_{h} h^{4} \\
& +t \phi+\frac{1}{2} m_{\phi 0}^{2} \phi^{2}+\frac{1}{3} a_{\phi} \phi^{3}+\frac{1}{4} \lambda_{\phi} \phi^{4} .
\end{aligned}
$$

In the limit of large Higgs mass, the Higgs- $\phi$ mixing is given by

$$
\sin \theta \simeq\left(\mu+\lambda_{h \phi} \phi\right) v / m_{h}^{2},
$$

where $m_{h}$ is a physical Higgs mass and $v=\langle h\rangle$ is its VEV. To derive the expression for the $\phi$ mass, let us integrate out the Higgs boson. This amounts for a substitution of $h^{2}$ solving $V_{h}^{\prime}=0$, namely,

$h^{2} \rightarrow \frac{1}{\lambda_{h}}\left\{m_{h 0}^{2}-\mu \phi-\frac{1}{2} \lambda_{h \phi} \phi^{2}\right\} \simeq v^{2}-\frac{\mu \phi}{\lambda_{h}}-\frac{\lambda_{h \phi}}{2 \lambda_{h}} \phi^{2}$,

into the scalar potential (A1). One can neglect the effect of $\phi$ kinetic term renormalization when working in the leading order in mixing. The resulting $\phi$ effective potential becomes

$$
\begin{aligned}
V(\phi)= & \phi\left\{t+\frac{1}{2} \mu v^{2}\right\} \\
& +\frac{1}{2} \phi^{2}\left\{m_{\phi 0}^{2}+\frac{\lambda_{h \phi}}{2} v^{2}-\frac{1}{2 \lambda_{h}} \mu^{2}\right\} \\
& +\frac{1}{3} \phi^{3}\left\{a_{\phi}-\frac{3 \lambda_{h \phi}}{4 \lambda_{h}} \mu\right\} \\
& +\frac{1}{4} \phi^{4}\left\{\lambda_{\phi}-\frac{\lambda_{h \phi}^{2}}{4 \lambda_{h}}\right\} \\
\equiv & \hat{t} \phi+\frac{1}{2} \hat{m}_{\phi 0}^{2} \phi^{2}+\frac{1}{3} \hat{a}_{\phi} \phi^{3}+\frac{1}{4} \hat{\lambda}_{\phi} \phi^{4} .
\end{aligned}
$$

By a $\phi$ field constant shift, one can always fix $\phi=0$ in the actual minimum. Such a redefinition will also shift the values of the parameters of the scalar potentials (A1), (A4), in particular the new coefficient of $\phi^{1}$ in (A4) has to vanish, $\hat{t}=0$. In a shifted theory, the $\phi$ mixing and mass read in terms of redefined parameters (for which we use the old notations)

$$
\begin{gathered}
\sin \theta \simeq \mu v / m_{h}^{2}, \\
m_{\phi}^{2} \simeq m_{\phi 0}^{2}+\frac{\lambda_{h \phi}}{2} v^{2}-\sin ^{2} \theta m_{h}^{2} .
\end{gathered}
$$

In the absence of fine tunings in the expression for the mass (A10), one expects that

$$
m_{\phi}^{2} \gtrsim \sin ^{2} \theta m_{h}^{2},
$$

which represents the so called naturalness bound. While the above derivation is based on tree-level quantities, an analogous bound (up to a loop factor) can also be obtained by requiring the quantum corrections to not exceed the physical $\phi$ mass; see Ref. [51]. One can also notice from Eq. (A10) that naturalness implies a bound on the crossquartic coupling $\lambda_{h \phi} \lesssim m_{\phi}^{2} / v^{2}$.

It is also possible to identify a generic condition under which the bound gets saturated. To this end it is convenient to work in the original basis of Eq. (A4), i.e., without $\phi$ shift, in order to keep an explicit dependence on the parameters of possibly different physical origin. A typical minimum of a polynomial potential is formed by a balance between two dominant terms with opposite slopes. We can approximately describe the potential around the relevant minimum with

$$
\begin{gathered}
V \simeq \frac{c_{p}}{p} \phi^{p}-\frac{c_{q}}{q} \phi^{q}, \\
\langle\phi\rangle \simeq\left(c_{p} / c_{q}\right)^{\frac{1}{q-p}}, \\
m_{\phi}^{2} \simeq(p-q) c_{p}^{\frac{q-2}{q-p}} c_{q}^{\frac{p-2}{p-q}} .
\end{gathered}
$$

We then notice that for $q=2$ the physical mass becomes independent of $c_{p}$ and is simply given by a single coefficient, $m_{\phi}^{2} \simeq c_{2}$, up to an order-one factor. Furthermore, for $\mu^{2} \gg 2 \lambda_{h} m_{\phi 0}^{2}+2 \lambda_{h} \lambda_{h \phi} v^{2}$ this single coefficient is uniquely related to $\mu$, by $m_{\phi}^{2} \simeq c_{2} \simeq \frac{1}{2 \lambda_{h}} \mu^{2}$ as follows from Eq. (A5). Substituting $\mu \simeq \sqrt{\lambda_{h}} m_{\phi}$ into the mixing (A2) we find that the first term in this expression saturates the naturalness bound, i.e.,

$$
\sin \theta \simeq m_{\phi} / m_{h} .
$$

This means that whenever (a) the quadratic term (A5) in the effective $\phi$ potential is dominated by the term $\propto \mu^{2}$ originating from the cubic interaction with the Higgs, and (b) the minimum is formed by a balance between the quadratic term and any other, the naturalness bound is saturated. 
[1] E. Aprile et al., Observation of excess electronic recoil events in XENON1T, Phys. Rev. D 102, 072004 (2020).

[2] D. Akerib et al. (LUX Collaboration), First Searches for Axions and Axionlike Particles with the LUX Experiment, Phys. Rev. Lett. 118, 261301 (2017).

[3] N. Viaux, M. Catelan, P. B. Stetson, G. Raffelt, J. Redondo, A. A. R. Valcarce, and A. Weiss, Neutrino and Axion Bounds from the Globular Cluster M5 (NGC 5904), Phys. Rev. Lett. 111, 231301 (2013).

[4] M. Giannotti, I. G. Irastorza, J. Redondo, A. Ringwald, and K. Saikawa, Stellar recipes for axion hunters, J. Cosmol. Astropart. Phys. 10 (2017) 010.

[5] B. M. Hansen, H. Richer, J. Kalirai, R. Goldsbury, S. Frewen, and J. Heyl, Constraining neutrino cooling using the hot white Dwarf luminosity function in the globular cluster 47 Tucanae, Astrophys. J. 809, 141 (2015).

[6] R. Budnik, O. Davidi, H. Kim, G. Perez, and N. Priel, Searching for a solar relaxion or scalar particle with XENON1T and LUX, Phys. Rev. D 100, 095021 (2019).

[7] F. Takahashi, M. Yamada, and W. Yin, XENON1T anomaly from anomaly-free ALP dark matter and its implications for stellar cooling anomaly, Phys. Rev. Lett. 125, 161801 (2020).

[8] K. Kannike, M. Raidal, H. Veermäe, A. Strumia, and D. Teresi, Dark Matter and the XENON1T electron recoil excess, Phys. Rev. D 102, 095002 (2020).

[9] G. Alonso-Álvarez, F. Ertas, J. Jaeckel, F. Kahlhoefer, and L. Thormaehlen, Hidden photon dark matter in the light of XENON1T and stellar cooling, J. Cosmol. Astropart. Phys. 11 (2020) 029.

[10] d. Amaral, D. W. Praia, D. G. Cerdeno, P. Foldenauer, and E. Reid, Solar neutrino probes of the muon anomalous magnetic moment in the gauged $U(1)_{L_{\mu}-L_{\tau}}$, J. High Energy Phys. 12 (2020) 155.

[11] B. Fornal, P. Sandick, J. Shu, M. Su, and Y. Zhao, Boosted dark matter interpretation of the XENON1T excess, Phys. Rev. Lett. 125, 161804 (2020).

[12] C. Boehm, D. G. Cerdeno, M. Fairbairn, P. A. Machado, and A. C. Vincent, Light new physics in XENON1T, Phys. Rev. D 102, 115013 (2020).

[13] A. Bally, S. Jana, and A. Trautner, Neutrino self-interactions and XENON1T electron recoil excess, Phys. Rev. Lett. 125, 161802 (2020).

[14] K. Harigaya, Y. Nakai, and M. Suzuki, Inelastic Dark Matter Electron Scattering and the XENON1T Excess, Phys. Lett. B 809, 135729 (2020).

[15] L. Su, W. Wang, L. Wu, J. M. Yang, and B. Zhu, Xenon1T anomaly: Inelastic cosmic ray boosted dark matter, Phys. Rev. D 102, 115028 (2020).

[16] M. Du, J. Liang, Z. Liu, V. Q. Tran, and Y. Xue, On-shell mediator dark matter models and the Xenon1T anomaly, Chin. Phys. C 45, 013114 (2021).

[17] L. Di Luzio, M. Fedele, M. Giannotti, F. Mescia, and E. Nardi, Solar axions cannot explain the XENON1T excess, Phys. Rev. Lett. 125, 131804 (2020).

[18] U. K. Dey, T. N. Maity, and T. S. Ray, Prospects of Migdal effect in the explanation of XENON1T electron recoil excess, Phys. Lett. B 811, 135900 (2020).
[19] Y. Chen, J. Shu, X. Xue, G. Yuan, and Q. Yuan, Sun heated MeV-scale Dark Matter and the XENON1T electron recoil excess, J. High Energy Phys. 04 (2021) 282.

[20] N. F. Bell, J. B. Dent, B. Dutta, S. Ghosh, J. Kumar, and J. L. Newstead, Explaining the XENON1T excess with luminous dark matter, Phys. Rev. Lett. 125, 161803 (2020).

[21] J. Buch, M. A. Buen-Abad, J. Fan, and J. S. C. Leung, Galactic origin of relativistic bosons and XENON1T excess, J. Cosmol. Astropart. Phys. 10 (2020) 051.

[22] G. Choi, M. Suzuki, and T. T. Yanagida, XENON1T anomaly and its implication for decaying warm dark matter, Phys. Lett. B 811, 135976 (2020).

[23] D. Aristizabal Sierra, V. De Romeri, L. Flores, and D. Papoulias, Light vector mediators facing XENON1T data, Phys. Lett. B 809, 135681 (2020).

[24] G. Paz, A. A. Petrov, M. Tammaro, and J. Zupan, Shining dark matter in Xenon1T, Phys. Rev. D 103, L051703 (2021).

[25] H. M. Lee, Exothermic dark matter for XENON1T excess, J. High Energy Phys. 01 (2021) 019.

[26] A. E. Robinson, XENON1T observes tritium, arXiv: 2006.13278.

[27] Q.-H. Cao, R. Ding, and Q.-F. Xiang, Exploring for sub$\mathrm{MeV}$ boosted dark matter from xenon electron direct detection, Chin. Phys. C 45, 045002 (2021).

[28] R. Primulando, J. Julio, and P. Uttayarat, Collider constraints on a dark matter interpretation of the XENON1T excess, Eur. Phys. J. C 80, 1084 (2020).

[29] A. N. Khan, Can nonstandard neutrino interactions explain the XENON1T spectral excess?, Phys. Lett. B 809, 135782 (2020).

[30] K. Nakayama and Y. Tang, Gravitational production of hidden photon dark matter in light of the XENON1T excess, Phys. Lett. B 811, 135977 (2020).

[31] Y. Jho, J.-C. Park, S. C. Park, and P.-Y. Tseng, Gauged lepton number and cosmic-ray boosted dark matter for the XENON1T excess, Phys. Lett. B 811, 135863 (2020).

[32] M. Baryakhtar, A. Berlin, H. Liu, and N. Weiner, Electromagnetic signals of inelastic dark matter scattering, arXiv:2006.13918.

[33] H. An, M. Pospelov, J. Pradler, and A. Ritz, New limits on dark photons from solar emission and $\mathrm{keV}$ scale dark matter, Phys. Rev. D 102, 115022 (2020).

[34] P. W. Graham, D. E. Kaplan, and S. Rajendran, Cosmological Relaxation of the Electroweak Scale, Phys. Rev. Lett. 115, 221801 (2015).

[35] T. Flacke, C. Frugiuele, E. Fuchs, R. S. Gupta, and G. Perez, Phenomenology of relaxion-Higgs mixing, J. High Energy Phys. 06 (2017) 050.

[36] K. Choi and S. H. Im, Constraints on relaxion windows, J. High Energy Phys. 12 (2016) 093.

[37] E. Aprile et al. (XENON Collaboration), Light Dark Matter Search with Ionization Signals in XENON1T, Phys. Rev. Lett. 123, 251801 (2019).

[38] E. Hardy and R. Lasenby, Stellar cooling bounds on new light particles: Plasma mixing effects, J. High Energy Phys. 02 (2017) 033.

[39] J. Redondo, Solar axion flux from the axion-electron coupling, J. Cosmol. Astropart. Phys. 12 (2013) 008. 
[40] N. Vinyoles, A. M. Serenelli, F. L. Villante, S. Basu, J. Bergström, M. Gonzalez-Garcia, M. Maltoni, C. Peña-Garay, and N. Song, A new generation of standard solar models, Astrophys. J. 835, 202 (2017).

[41] F. T. Avignone, R. Brodzinski, S. Dimopoulos, G. Starkman, A. Drukier, D. Spergel, G. Gelmini, and B. Lynn, Laboratory limits on solar axions from an ultralow background germanium spectrometer, Phys. Rev. D 35, 2752 (1987).

[42] F. Alessandria et al. (CUORE Collaboration), Search for $14.4 \mathrm{keV}$ solar axions from M1 transition of Fe-57 with CUORE crystals, J. Cosmol. Astropart. Phys. 05 (2013) 007.

[43] M. Pospelov, A. Ritz, and M. B. Voloshin, Bosonic superWIMPs as keV-scale dark matter, Phys. Rev. D 78, 115012 (2008).

[44] E. Aprile et al. (XENON Collaboration), Energy resolution and linearity in the $\mathrm{keV}$ to $\mathrm{MeV}$ range measured in XENON1T, Eur. Phys. J. C 80, 785 (2020).

[45] G. B. Gelmini, V. Takhistov, and E. Vitagliano, Scalar direct detection: In-medium effects, Phys. Lett. B 809, 135779 (2020).

[46] G. Cowan, K. Cranmer, E. Gross, and O. Vitells, Asymptotic formulae for likelihood-based tests of new physics, Eur. Phys. J. C 71, 1554 (2011); Erratum, Eur. Phys. J. C 73, 2501 (2013).

[47] F. Piazza and M. Pospelov, Sub-eV scalar dark matter through the super-renormalizable Higgs portal, Phys. Rev. D 82, 043533 (2010).

[48] A. Arvanitaki, S. Dimopoulos, and K. Van Tilburg, Sound of Dark Matter: Searching for Light Scalars with ResonantMass Detectors, Phys. Rev. Lett. 116, 031102 (2016).

[49] P. W. Graham, D. E. Kaplan, J. Mardon, S. Rajendran, and W. A. Terrano, Dark matter direct detection with accelerometers, Phys. Rev. D 93, 075029 (2016).

[50] G. Raffelt, Stars as laboratories for fundamental physics: The astrophysics of neutrinos, axions, and other weakly interacting particles (1996).

[51] A. Banerjee, H. Kim, O. Matsedonskyi, G. Perez, and M. S. Safronova, Probing the relaxed relaxion at the luminosity and precision frontiers, J. High Energy Phys. 07 (2020) 153.

[52] J. Grifols, E. Masso, and S. Peris, Energy loss from the Sun and red giants: Bounds on short range baryonic and leptonic forces, Mod. Phys. Lett. A 04, 311 (1989).

[53] G. Raffelt, Limits on a $C P$-violating scalar axion-nucleon interaction, Phys. Rev. D 86, 015001 (2012).

[54] M. S. Turner, Axions from SN 1987a, Phys. Rev. Lett. 60, 1797 (1988).

[55] J. A. Frieman, S. Dimopoulos, and M. S. Turner, Axions and stars, Phys. Rev. D 36, 2201 (1987).

[56] A. Burrows, M. S. Turner, and R. Brinkmann, Axions and SN 1987a, Phys. Rev. D 39, 1020 (1989).

[57] N. Bar, K. Blum, and G. D'amico, Is there a supernova bound on axions?, Phys. Rev. D 101, 123025 (2020).

[58] E. M. Burbidge, G. R. Burbidge, W. A. Fowler, and F. Hoyle, Synthesis of the elements in stars, Rev. Mod. Phys. 29, 547 (1957).

[59] W. A. Fowler and F. Hoyle, Neutrino processes and pair formation in massive stars and supernovae, Astrophys. J. Suppl. Ser. 9, 201 (1964).
[60] F. Hoyle and W. A. Fowler, Nucleosynthesis in Supernovae, Astrophys. J. 132, 565 (1960); Erratum, 134, 1028 (1961).

[61] D. Kushnir and B. Katz, Failure of a neutrino-driven explosion after core-collapse may lead to a thermonuclear supernova, Astrophys. J. 811, 97 (2015).

[62] V. Khachatryan et al. (CMS Collaboration), Search for a standard model-like Higgs boson in the mu+muand e+e- decay channels at the LHC, Phys. Lett. B 744, 184 (2015).

[63] W. Altmannshofer, J. Brod, and M. Schmaltz, Experimental constraints on the coupling of the Higgs boson to electrons, J. High Energy Phys. 05 (2015) 125.

[64] A. Dery, C. Frugiuele, and Y. Nir, Large Higgs-electron Yukawa coupling in 2HDM, J. High Energy Phys. 04 (2018) 044.

[65] D. B. Kaplan, A. E. Nelson, and N. Weiner, Neutrino Oscillations as a Probe of Dark Energy, Phys. Rev. Lett. 93, 091801 (2004).

[66] A. Hook and J. Huang, Probing axions with neutron star inspirals and other stellar processes, J. High Energy Phys. 06 (2018) 036.

[67] R. Balkin, J. Serra, K. Springmann, and A. Weiler, The QCD axion at finite density, J. High Energy Phys. 07 (2020) 221.

[68] A. Hook and G. Marques-Tavares, Relaxation from particle production, J. High Energy Phys. 12 (2016) 101.

[69] K. Choi, H. Kim, and T. Sekiguchi, Dynamics of the cosmological relaxation after reheating, Phys. Rev. D 95, 075008 (2017).

[70] W. Tangarife, K. Tobioka, L. Ubaldi, and T. Volansky, Dynamics of relaxed inflation, J. High Energy Phys. 02 (2018) 084.

[71] M. Ibe, Y. Shoji, and M. Suzuki, Fast-rolling relaxion, J. High Energy Phys. 11 (2019) 140.

[72] K. Kadota, U. Min, M. Son, and F. Ye, Cosmological relaxation from dark fermion production, J. High Energy Phys. 02 (2020) 135.

[73] N. Fonseca, E. Morgante, R. Sato, and G. Servant, Axion fragmentation, J. High Energy Phys. 04 (2020) 010 .

[74] N. Fonseca, E. Morgante, R. Sato, and G. Servant, Relaxion fluctuations (self-stopping relaxion) and overview of relaxion stopping mechanisms, J. High Energy Phys. 05 (2020) 080.

[75] N. Blinov, S. A. Ellis, and A. Hook, Consequences of finetuning for fifth force searches, J. High Energy Phys. 11 (2018) 029.

[76] A. Hook and J. Huang, Searches for other vacua. Part I. Bubbles in our universe, J. High Energy Phys. 08 (2019) 148.

[77] S. Alighanbari, G. S. Giri, F. L. Constantin, V. I. Korobov, and S. Schiller, Precise test of quantum electrodynamics and determination of fundamental constants with $\mathrm{hd}^{+}$ions, Nature (London) 581, 152 (2020).

[78] H. Leeb and J. Schmiedmayer, Constraint on Hypothetical Light Interacting Bosons from Low-Energy Neutron Experiments, Phys. Rev. Lett. 68, 1472 (1992). 
[79] V. Nesvizhevsky, G. Pignol, and K. Protasov, Neutron scattering and extra short range interactions, Phys. Rev. D 77, 034020 (2008).

[80] Y. Pokotilovski, Constraints on new interactions from neutron scattering experiments, Phys. At. Nucl. 69, 924 (2006).

[81] C. Frugiuele, E. Fuchs, G. Perez, and M. Schlaffer, Constraining new physics models with isotope shift spectroscopy, Phys. Rev. D 96, 015011 (2017).
[82] W. DeRocco, P. W. Graham, and S. Rajendran, Exploring the robustness of stellar cooling constraints on light particles, Phys. Rev. D 102, 075015 (2020).

[83] A. D. Linde, Particle physics and inflationary cosmology, Contemp. Concepts Phys. 5, 1 (1990).

[84] G. W. Anderson and L.J. Hall, The electroweak phase transition and baryogenesis, Phys. Rev. D 45, 2685 (1992). 